\title{
Régénération osseuse en terrain irradié
}

\author{
Weiss P, Malard O \\ Laboratoire d'Ingénierie tissulaire ostéo-articulaire et dentaire INSERM U791 \\ Faculté d'Odontologie, Nantes, France
}

Le cancer des VADS représente le 4ème cancer chez l'homme en France et ce cancer a le taux de mortalité le plus élevé en Europe ; la France est le 3ème pays le plus touché dans le monde.

La cavité buccale constitue l'une des principales localisations des cancers des VADS et son incidence est l'une des plus élevées dans le monde.

Les cancers de la cavité buccale sont traités dans la grande majorité des cas par l'association chirurgie - radiothérapie, responsable de séquelles dento-squelettiques et orofaciales dont les conséquences fonctionnelles (mastication, déglutition, alimentation, phonation), esthétiques, psychologiques et sociales hypothèquent de manière significative la qualité de vie des patients.

Les deux tiers de ces cancers sont diagnostiqués à un stade avancé, III ou IV, avec le plus souvent extension à la mandibule et envahissement ganglionnaire. L'exérèse chirurgicale peut comporter une exérèse partielle, segmentaire ou non, de la mandibule avec perte de dents et trouble de l'articulé dentaire. Le traitement et le pronostic dépendent entre autres des possibilités d'exérèse chirurgicale et donc des possibilités de réhabilitation post-chirurgicale. La radiothérapie entraine également des complications et des séquelles dentaires et osseuses. L'ostéoradionécrose mandibulaire constitue la complication post-radique la plus grave et elle peut mettre en jeu le pronostic vital ; il n'existe aucun traitement réellement curatif. Les troubles trophiques, secondaires à la chirurgie et la radiothérapie, réduisent considérablement les possibilités de réhabilitation dentaire et osseuse ainsi que le traitement de l'ostéoradionécrose.

Après avoir réalisé plusieurs études chez l'animal pour obtenir un modèle d'os irradié, nous avons constaté que les biomatériaux ou la moelle osseuse seule étaient incapables de régénérer de l'os en territoire irradié chez l'animal. Les cellules souches mésenchymateuses issues de la moelle associées à des biomatériaux ne permettent pas non plus de régénérer du tissu osseux dans cette indication. Seule l'association de biomatériaux à type de phosphates de calcium biphasés (MBCP) avec de la moelle osseuse totale a montré une réelle efficacité.

Dans le cadre d'une étude clinique de phase I, 10 patients présentant une ostéoradionécrose débutante ont été traités par l'association de granules de MBCP avec de la moelle osseuse totale autologue : les résultats préliminaires sont encourageants sur les premiers 5 patients traités.

Correspondance: pweiss@sante.univ-nantes.fr 